\title{
Ideals induced by Tsirelson submeasures
}

\author{
by
}

Ilijas F a r ah (North York, Ont.)

\begin{abstract}
We use Tsirelson's Banach space ([2]) to define an $F_{\sigma}$ P-ideal which refutes a conjecture of Mazur and Kechris (see $[12,9,8]$ ).
\end{abstract}

1. Introduction. By the dichotomy results of Silver and HarringtonKechris-Louveau (see $[10,8]$ ), the Borel-cardinality of quotients over Borel equivalence relations on Polish spaces is well-understood below $\mathcal{P}(\mathbb{N}) /$ Fin. This cannot be said for the next level of this ordering, even if we restrict our attention to Borel-cardinalities of quotients $\mathcal{P}(\mathbb{N}) / \mathcal{I}$ over Borel ideals $\mathcal{I}$. The two natural "successors" of Fin are the Fubini ideals on $\mathbb{N}^{2}$ : Fin $\times \emptyset$ (also called $I_{1}$ ) consisting of all sets with only finitely many nonempty vertical sections, and $\emptyset \times$ Fin (also called $I_{3}$ and Fin ${ }^{\omega}$ ) consisting of all sets all of whose vertical sections are finite. By results of Solecki ([17]), quotients over these two ideals are the critical points for quotients over Borel ideals which are not P-ideals and for Borel P-ideals which are not $F_{\sigma}$, respectively ( $\mathcal{I}$ is a $P$-ideal if it is $\sigma$-directed under the inclusion modulo finite). In [9], Kechris posed the following trichotomy conjecture for Borel ideals $\mathcal{I}$ such that $\mathcal{P}(\mathbb{N}) / \mathcal{I} \mathbb{Z}_{\mathrm{B}} \mathcal{P}(\mathbb{N}) /$ Fin: at least one of $\mathcal{P}\left(\mathbb{N}^{2}\right) /$ Fin $\times \emptyset$, $\mathcal{P}\left(\mathbb{N}^{2}\right) / \emptyset \times$ Fin, and $\mathcal{P}(\mathbb{N}) / \mathcal{I}_{1 / n}$ is $\leq_{\mathrm{B}} \mathcal{P}(\mathbb{N}) / \mathcal{I}$ (the summable ideal $\mathcal{I}_{1 / n}$ is defined below). By the above results of Solecki, this is equivalent to an earlier dichotomy conjecture of Mazur ([12]): If $\mathcal{I}$ is an $F_{\sigma}$ ideal such that $\mathcal{P}(\mathbb{N}) / \mathcal{I} \quad \mathbb{B}_{\mathrm{B}} \mathcal{P}(\mathbb{N}) /$ Fin, then either $\mathcal{P}\left(\mathbb{N}^{2}\right) /$ Fin $\times \emptyset$ or $\mathcal{P}(\mathbb{N}) / \mathcal{I}_{1 / n}$ is $\leq_{\mathrm{B}} \mathcal{P}(\mathbb{N}) / \mathcal{I}$.

1991 Mathematics Subject Classification: 04A15, 46B.

I would like to thank S. Todorčević for giving me several useful suggestions while I was working on this problem, to K. Mazur who has noticed quite a few inaccuracies in the original version of this paper, and to an anonymous referee whose remarks have considerably simplified the presentation, in particular in $\S 6$.

Supported by NSERC of Canada. 
Consider an ordering on Borel ideals simpler than $\leq_{\mathrm{B}}$ :

$\mathcal{I} \leq_{\mathrm{RB}}^{+} \mathcal{J}$ if there is $A \subseteq \mathbb{N}$ and $h: A \rightarrow \mathbb{N}$

$$
\text { such that } B \in \mathcal{I} \text { iff } h^{-1}(B) \in \mathcal{J} \text {. }
$$

If $A=\mathbb{N}$, then we write $\mathcal{I} \leq_{\mathrm{RB}} \mathcal{J}$. Clearly, $\mathcal{I} \leq_{\mathrm{RB}}^{+} \mathcal{J}$ implies $\mathcal{P}(\mathbb{N}) / \mathcal{I} \leq_{\mathrm{B}}$ $\mathcal{P}(\mathbb{N}) / \mathcal{J}$, as the mapping $A \mapsto h^{-1}(A)$ verifies. It is rather surprising that the converse is often true; for example, the above Solecki dichotomy results are proved for the $\leq_{\mathrm{RB}}$-ordering (see also Lemma 2.1 below).

Any $\mathcal{I}$ serving as a counterexample to the Kechris-Mazur conjecture, or KMC, would have to be an $F_{\sigma}$ P-ideal. Until recently, the only known $F_{\sigma}$ P-ideals were the summable ideals, that is, ones of the form

$$
\mathcal{I}_{f}=\left\{A: \nu_{f}(A)<\infty\right\}=\left\{A: \lim _{n}\left(\nu_{f}(A \backslash n)\right)=0\right\}
$$

where $\nu_{f}(A)=\sum_{n \in A} f(n)$ for some $f: \mathbb{N} \rightarrow \mathbb{R}^{+}$. These ideals cannot serve as a counterexample to the Kechris-Mazur conjecture, since we have either $\mathcal{I}_{f} \leq_{\mathrm{RB}}^{+}$Fin or $\mathcal{I}_{1 / n} \leq_{\mathrm{RB}}^{+} \mathcal{I}_{f}$ (note that this is false for the $\leq_{\mathrm{RB}}$ ordering, and this is why we introduce $\leq_{\mathrm{RB}}^{+}$). By [17, Theorem 3.3] (see also [11, Lemma $1.2])$, all $F_{\sigma}$ P-ideals are of the form

$$
\mathcal{I}=\{A: \phi(A)<\infty\}=\left\{A: \lim _{i} \phi(A \backslash i)=0\right\}
$$

for some lower semicontinuous submeasure $\phi$, i.e. a mapping such that $\phi(A) \leq \phi(A \cup B) \leq \phi(A)+\phi(B), \phi(\emptyset)=0$ and $\lim _{i} \phi_{i}(A \cap i)=\phi(A)$ for all $A, B$. The first non-summable $F_{\sigma}$ P-ideals were discovered in [4] (see also [3]). All these ideals were of the form

$$
\mathcal{I}_{\left\{\phi_{n}\right\}}=\left\{A: \sum_{i} \phi_{i}(A)<\infty\right\},
$$

where for some sequence $\left\{n_{i}\right\}$ each $\phi_{i}$ is a submeasure on the interval $\left[n_{i}, n_{i+1}\right)$. But such ideals satisfy the KMC since if $\lim _{i} \sup _{j} \phi_{i}(\{j\})=0$ (and this can be assumed without loss of generality by going to a positive set) then there are $s_{i}$ and $m_{i}$ such that

$$
\phi_{k}\left(s_{i}\right) \begin{cases}\approx 1 / i, & k=m_{i}, \\ =0, & \text { otherwise }\end{cases}
$$

so that the map collapsing $s_{i}$ to $i$ witnesses $\mathcal{I}_{1 / n} \leq_{\mathrm{RB}}^{+} \mathcal{I}_{\left\{\phi_{i}\right\}}$. An $F_{\sigma}$ P-ideal which is not of the form $\mathcal{I}_{\left\{\phi_{i}\right\}}$ was later found by Solecki ([16]), who also proved that this ideal is of the form $\mathcal{I}_{\left\{\phi_{i}\right\}}$ when restricted to a positive set, so it is again $\geq_{\mathrm{RB}}^{+} \mathcal{I}_{1 / n}$. Another class of $F_{\sigma}$ P-ideals, suggested by Kechris, are ideals of the form

$$
\mathcal{I}=\left\{A: \sqrt[p]{\left.\sum_{i} \phi_{i}(A)\right)^{p}}<\infty\right\}
$$


for a sequence of submeasures $\phi_{i}$ as before and $p>1$, but these again do not serve as a counterexample to KMC, for the same reason as $\mathcal{I}_{\left\{\phi_{i}\right\}}$. (However, using methods and results of [7] it can be proved that the Borel-cardinalities of these quotients are different for different $p$ 's.)

The new $F_{\sigma}$ P-ideal which we define here is extracted from Tsirelson space, an infinite-dimensional Banach space which does not contain a copy of $c_{0}$ or any $\ell_{p}$ (see [2]). The study of this space has played a prominent role in the recent striking developments in the theory of infinite-dimensional Banach spaces (see [6], [13, p. 956]). It is likely that other Banach spaces will give rise to interesting examples of analytic P-ideals (see [5]).

After the completion of this paper, we have learned that our main result, Theorem 3.1, was independently proved by B. Veličković ([19]).

The paper is organized as follows. In $\S 2$ we prove that $\mathcal{P}(\mathbb{N}) / \mathcal{I}_{1 / n} \leq{ }_{\mathrm{B}}$ $\mathcal{P}(\mathbb{N}) / \mathcal{I}$ is equivalent to $\mathcal{I}_{1 / n} \leq_{\mathrm{RB}}^{+} \mathcal{I}$. In $\S 3$ we introduce the ideals $\mathcal{T}_{f h}$. In $\S \S 4-6$ various properties of these ideals are proved, and in $\S 7$ we conclude the proof that $\mathcal{P}(\mathbb{N}) / \mathcal{T}_{f h}$ serves as a counterexample to the Kechris-Mazur conjecture.

A word on notation: If $s, t$ are finite sets of integers and $n$ is an integer, by $s<t$ we denote the fact that $\max s<\min t$, and by $n<s(n>s)$ the fact that $n<\min s(n>\max s$, respectively).

The results of this note were presented at the XI Latinamerican Logic Symposium in Merida, Venezuela, in July 1998. I would like to thank the organizers, in particular Carlos Uzcategui for his warm hospitality.

2. The first reduction. A quotient $\mathcal{P}(\mathbb{N}) / \mathcal{I}$ has smaller Borel-cardinality than the quotient $\mathcal{P}(\mathbb{N}) / \mathcal{J}$ (in symbols $\left.\mathcal{P}(\mathbb{N}) / \mathcal{I} \leq_{\mathrm{B}} \mathcal{P}(\mathbb{N}) / \mathcal{J}\right)$ if there is a Borel mapping $F: \mathcal{P}(\mathbb{N}) \rightarrow \mathcal{P}(\mathbb{N})$ such that $X \triangle Y \in \mathcal{I}$ iff $F(X) \triangle F(Y) \in \mathcal{J}$.

In the following lemma it is proved that the $\mathrm{KMC}$ is equivalent to its apparently stronger version, appearing in [4], which states: For every analytic ideal $\mathcal{I}$ such that $\mathcal{I} \mathbb{Z}_{\mathrm{RB}}$ Fin one of Fin $\times \emptyset, \emptyset \times$ Fin or $\mathcal{I}_{1 / n}$ is $\leq_{\mathrm{RB}} \mathcal{I} \mid A$ for some $\mathcal{I}$-positive $A$. (It is well-known that $\mathcal{P}(\mathbb{N}) / \mathcal{I} \leq_{\mathrm{B}} \mathcal{P}(\mathbb{N}) /$ Fin is equivalent to $\mathcal{I} \leq_{\mathrm{RB}}$ Fin.)

Lemma 2.1. If $\mathcal{J}$ is an analytic P-ideal such that $\mathcal{P}(\mathbb{N}) / \mathcal{I}_{1 / n} \leq_{\mathrm{B}} \mathcal{P}(\mathbb{N}) / \mathcal{J}$, then $\mathcal{I}_{1 / n} \leq_{\mathrm{RB}}^{+} \mathcal{J}$. Moreover, there are $w_{1}<w_{2}<\ldots$ in Fin such that the map collapsing $w_{i}$ to $i$ witnesses this.

Proof. By [17], we can fix a lower semicontinuous submeasure $\phi$ such that $\mathcal{J}=\left\{A: \lim _{i} \phi(A \backslash i)=0\right\}$. Let $F: \mathcal{P}(\mathbb{N}) \rightarrow \mathcal{P}(\mathbb{N})$ be a Borel reduction. By a standard use of stabilizers, similar to the one below, we can assume that $F$ is continuous (see also [18]). Find integers $1=a_{1}<b_{1}<a_{2}<b_{2}<\ldots$, $s_{i} \subseteq\left[b_{i}, a_{i+1}\right)$ and $k_{i} \in\left(b_{i}, a_{i+1}\right)$ so that for all $i$, all $u, v \subseteq b_{i}$, and all $X, Y \subseteq\left[a_{i+1}, \infty\right)$ : 
(1) $a_{i}>2^{i}, b_{i}>2 a_{i}$,

(2) $\left(F\left(u \cup s_{i} \cup X\right) \triangle F\left(u \cup s_{i} \cup Y\right)\right) \cap k_{i}=\emptyset$,

(3) $\phi\left(\left(F\left(u \cup s_{i} \cup X\right) \triangle F\left(v \cup s_{i} \cup X\right)\right) \backslash k_{i}\right) \leq 2^{-i}$.

The method for construction of these sequences is standard, dating back to [15] and [18]: Assume that $a_{i}, b_{i}(i \leq n)$ and $s_{j}, k_{j}(j \leq n-1)$ as above have been chosen, but there are no $s_{n}, k_{n}$ and $a_{n+1}$ satisfying the requirements. Condition (1) is easy to satisfy and since $F$ is continuous, (2) will be satisfied for every choice of $s_{n}$, a large enough $k_{n}$ and a large enough $a_{n+1}$. Therefore we can construct a sequence $b_{n}<t_{1}<l_{1}<t_{2}<l_{2}<\ldots$ so that $l_{i} \in \mathbb{N}$, $t_{i} \in$ Fin, and for all $i$ there are $u_{i}, v_{i} \subseteq a_{n}$ such that

$$
\phi\left(\left(F\left(u_{i} \cup \bigcup_{j=1}^{i} t_{j} \cup t_{i+1}\right) \triangle F\left(u_{i} \cup \bigcup_{j=1}^{i} t_{j} \cup t_{i+1}\right)\right) \backslash l_{i}\right)>2^{-n} .
$$

Pick $u, v$ such that $\langle u, v\rangle=\left\langle u_{i}, v_{i}\right\rangle$ infinitely often. Then $F\left(u \cup \bigcup_{i} t_{i}\right) \triangle$ $F\left(v \cup \bigcup_{i} t_{i}\right)$ is not in $\mathcal{J}$-a contradiction.

Assume $a_{n}, b_{n}, s_{n}$ and $k_{n}$ are chosen to satisfy the above conditions. By (1), $\nu_{1 / n}\left(\left[a_{i}, b_{i}\right]\right)=\sum_{j=a_{i}}^{b_{i}} 1 / j \geq 1 / n$ and there is $u_{i} \subseteq\left(a_{i}, b_{i}\right)$ such that $\left|\nu_{1 / n}\left(u_{i}\right)-1 / i\right| \leq 2^{-i}$ for every $i$. Let $C=\bigcup_{i} s_{i}$ and define $F_{1}$ : $P\left(\bigcup_{i}\left[a_{i}, b_{i}\right)\right) \rightarrow \mathcal{P}(\mathbb{N})$ by

$$
F_{1}(B)=F(B \cup C) \triangle F(C) .
$$

Then $F_{1}(\emptyset)=\emptyset$ and for $X, Y \subseteq \bigcup_{i}\left[a_{i}, b_{i}\right)$ we have $(X \cup C) \triangle(Y \cup C)=$ $X \triangle Y \in \mathcal{I}_{1 / n}$ iff $F_{1}(X) \triangle F_{1}(Y)=F(X \cup C) \triangle F(Y \cup C) \in \mathcal{J}$. By (2)-(3), for all $i$, all $u, v \subseteq \bigcup_{i \leq m}\left[a_{i}, b_{i}\right)$, and all $X, Y \subseteq \bigcup_{i \geq m+1}\left[a_{i}, b_{i}\right)$ we have:

(4) $\left(F_{1}(u \cup X) \triangle F_{1}(u \cup Y)\right) \cap k_{m}=\emptyset$,

(5) $\phi\left(\left(F_{1}(u \cup X) \triangle F_{1}(v \cup X)\right) \backslash k_{m}\right) \leq 2^{-m}$.

Let $w_{i}=F_{1}\left(u_{i}\right) \cap\left[k_{i-1}, k_{i}\right)$. Then a map collapsing $w_{i}$ to $i$ witnesses $\mathcal{I}_{1 / n} \leq_{\mathrm{RB}}^{+} \mathcal{J}$. This is implied by the following computations (assume $m \in A$ and let $\left.u_{X}=\bigcup_{i \in X} u_{i}\right)$ :

$$
\begin{aligned}
& t_{m}=\left(F_{1}\left(u_{A}\right) \triangle w_{A}\right) \cap\left[k_{m-1}, k_{m}\right) \\
& \left.\subseteq\left(F_{1}\left(u_{A}\right) \triangle F_{1}\left(u_{A \backslash m}\right)\right) \cup\left(F_{1}\left(u_{A \backslash m}\right) \triangle F_{1}\left(u_{m}\right)\right)\right) \cap\left[k_{m-1}, k_{m}\right) \\
& \text { (since } w_{A} \cap\left[k_{m-1}, k_{m}\right)=w_{m} \text { ) } \\
& \subseteq\left(\left(\left(F_{1}\left(u_{A}\right) \triangle F_{1}\left(u_{A \backslash m}\right)\right) \backslash a_{m}\right) \cup\left(F_{1}\left(u_{A \backslash m}\right) \triangle F_{1}\left(u_{m}\right)\right)\right) \cap k_{m} \\
& \text { (by (4) and (5), since } u_{A} \triangle u_{A \backslash m} \subseteq \bigcup_{i=1}^{m-1}\left[a_{i}, b_{i}\right) \\
& \text { and } \left.u_{A \backslash m} \triangle u_{m} \subseteq \bigcup_{i=m+1}^{\infty}\left[a_{i}, b_{i}\right)\right) \\
& =\left(F_{1}\left(u_{A}\right) \triangle F_{1}\left(u_{A \backslash m}\right)\right) \backslash a_{m},
\end{aligned}
$$

and therefore $\phi\left(t_{m}\right) \leq 2^{-m+1}$ if $m \in A$. An analogous computation shows 
that $\phi\left(t_{m}\right) \leq 2^{-m+1}$ also in the case when $m \notin A$, and therefore we have

$$
F_{1}\left(u_{A}\right) \triangle w_{A} \subseteq \bigcup_{m} t_{m} \cup\left[1, k_{1}\right) \in \mathcal{J} .
$$

To prove the moreover part, that we can assume that $w_{1}<w_{2}<\ldots$, find $1<n_{1}<m_{1}<n_{2}<m_{2}<\ldots$ so that $n_{i}>2^{i}, \nu_{1 / n}\left(\left[n_{i}, m_{i}\right]\right)-1 / i \mid \leq 2^{-i}$, and

$$
\max \bigcup_{j \in\left[n_{i}, m_{i}\right]} w_{j}<\min \bigcup_{j \in\left[n_{i+1}, m_{i+1}\right]} w_{j} .
$$

Then the sets $w_{i}^{\prime}=\bigcup_{j \in\left[n_{i}, m_{i}\right]} w_{j}$ are as required.

Let us digress a little and note that $\emptyset \times$ Fin also shares the nice property of $\mathcal{I}_{1 / n}$ from Lemma 2.1, as its proof readily shows.

Lemma 2.2. If $\mathcal{J}$ is an analytic P-ideal such that $\mathcal{P}\left(\mathbb{N}^{2}\right) / \emptyset \times$ Fin $\leq_{\mathrm{B}}$ $\mathcal{P}(\mathbb{N}) / \mathcal{J}$, then $\emptyset \times$ Fin $\leq_{\mathrm{RB}}^{+} \mathcal{J}\left\lceil A\right.$ for some $A \in \mathcal{J}^{+}$.

It would be interesting to find more ideals with this property shared by $\mathcal{I}_{1 / n}$ and $\emptyset \times$ Fin, since it considerably simplifies some questions about the Borel-cardinality of their quotients. Let us note that a pathological $F_{\sigma \delta} \mathrm{P}-$ ideal $\mathcal{J}$ constructed in $[3, \S 6]$ does not have this property. Namely, by a result of M. R. Oliver ([14]), $E_{\mathcal{J}}$ is Borel-reducible to $E_{\mathcal{Z}_{0}}$, the equivalence relation induced by the density zero ideal. But the ideal $\mathcal{Z}_{0}$ is nonpathological (see $[3]$ ), and therefore by [3, Proposition 6.5], $\mathcal{J} \leq_{\mathrm{RB}}^{+} \mathcal{Z}_{0}$ would imply that $\mathcal{J}$ is nonpathological as well.

3. Tsirelson submeasures and ideals. Assume that $\left\{x_{n}\right\}$ is an unconditional basic sequence in a Banach space $X$ such that $\lim _{n}\left\|\sum_{i=1}^{n} x_{i}\right\|=\infty$. Then

$$
\mathcal{J}=\left\{A:\left\|\sum_{n \in A} x_{n}\right\|<\infty\right\}
$$

is an analytic P-ideal, which we call a generalized summable ideal. Many analytic P-ideals are of this form, and ideals $\mathcal{T}_{f h}$ defined below are obtained in this way from the Tsirelson space, a Banach space which does not include a copy of $c_{0}$ or any $\ell_{p}$ (see [2]).

For sets $A, B \subseteq \mathbb{N}$, we often denote by $A B$ their intersection, $A \cap B$. Fix functions $f: \mathbb{N} \rightarrow \mathbb{R}$ and an increasing $h: \mathbb{N} \rightarrow \mathbb{N}$. A tuple

$$
\left\langle k, E_{1}, \ldots, E_{m}\right\rangle
$$

is $h$-admissible if $k \in \mathbb{N}, E_{i} \in$ Fin for all $i, k<E_{1}<E_{2}<\ldots<E_{m}$, and $m \leq h(k)$. We abbreviate tuples $\left\langle k, E_{1}, \ldots, E_{m}\right\rangle$ as $\langle k, \vec{E}\rangle$ and write $m=|\vec{E}|$, so that the necessary condition for the admissibility is $|\vec{E}| \leq h(k)$. Let $\mathcal{A}_{h}$ be the set of all $h$-admissible tuples. Define a sequence of Tsirelson 
submeasures $\tau_{n}=\tau_{f, h, n}(n \in \mathbb{N} \cup\{\infty\})$ as follows:

$$
\begin{aligned}
\tau_{f, h, 0}(A) & =\sup _{n \in A} f(n), \\
\tau_{f, h, n}^{k}(A) & =\sup _{\langle k, \vec{E}\rangle \in \mathcal{A}_{h}} \sum_{i=1}^{|\vec{E}|} \tau_{f, h, n}\left(E_{i} A\right), \\
\tau_{f, h, n+1}(A) & =\max \left\{\tau_{f, h, n}(A), \frac{1}{2} \sup _{k} \tau_{f, h, n}^{k}(A)\right\}, \\
\tau_{f, h, \infty}(A) & =\sup _{n} \tau_{f, h, n}(A)=\lim _{n} \tau_{f, h, n}(A) .
\end{aligned}
$$

We always omit $\infty$ in subscripts, so that $\tau_{f, h}$ stands for $\tau_{f, h, \infty}$ and $\tau_{f, h}^{k}$ stands for $\tau_{f, h, \infty}^{k}$. Similarly, when $f, h$ are clear from the context we write $\tau_{n}$ instead of $\tau_{f, h, n}, \tau^{k}$ instead of $\tau_{f, h}^{k}$, and so on. The submeasure $\tau=\tau_{f, h}$ defines a Tsirelson ideal, $\mathcal{T}_{f h}$, on $\mathbb{N}$ by

$$
\mathcal{T}_{\text {fh }}=\operatorname{Exh}(\tau)=\left\{A: \lim _{i} \tau(A \backslash i)=0\right\} .
$$

We always assume that $f: \mathbb{N} \rightarrow \mathbb{R}^{+}, \lim _{i} f(i)=0$, and $h$ is strictly increasing, unless otherwise specified.

TheOrem 3.1. Assume $f, h$ are as above. Then $\mathcal{P}(\mathbb{N}) / \mathcal{I}_{1 / n} \mathbb{Z}_{\mathrm{B}} \mathcal{P}(\mathbb{N}) / \mathcal{T}_{\text {fh }}$ and $\mathcal{T}_{f h}$ is an $F_{\sigma}$ P-ideal.

The proof of Theorem 3.1 occupies the rest of this note.

Lemma 3.2. Assume $f, h$ are as above. Then

(1) Either $\tau(A)=\sup _{i \in A} f(i)$ or $\tau(A)=\frac{1}{2} \sup _{k} \tau^{k}(A)$.

(2) All $\tau_{n}$ and $\tau$ are lower semicontinuous submeasures.

(3) $\tau(A)<\infty$ if and only if $\lim _{n} \tau(A \backslash n)=0$.

(4) $\tau_{m+1}(A)<\infty$ if and only if $\lim _{n} \tau_{m}(A \backslash n)=0$.

(5) $\mathcal{T}_{f h}=\{A \mid \tau(A)<\infty\}$ and therefore it is an $F_{\sigma}$ P-ideal.

Pr o o f. To prove (1), note that if $\tau(A)>\sup _{i \in A} f(i)$ then we have

$$
\tau(A)=\sup _{n}\left(\frac{1}{2} \sup _{k} \tau_{n}^{k}(A)\right)=\frac{1}{2} \sup _{k} \tau^{k}(A) .
$$

Statement (2) is obvious from the definition. In (3) only the direct implication requires a proof. Assume that $\lim _{n} \tau(A \backslash n) \neq 0$; then we can find $\varepsilon>0$ and finite sets $w_{1}<w_{2}<\ldots$ included in $A$ such that $\tau\left(w_{n}\right) \geq \varepsilon$ for all $n$. Fix $p \in \mathbb{N}$ and find $\bar{n}$ such that

$$
\min \left(w_{\bar{n}}\right)>2 p
$$

Then $A_{0}=\bigcup_{i=\bar{n}}^{\bar{n}+2 p} w_{i} \subseteq A$ and by (1) we get

$$
\tau\left(A_{0}\right) \geq \frac{1}{2} \sum_{i=\bar{n}}^{\bar{n}+2 p} \tau\left(w_{i}\right) \geq p \varepsilon .
$$

Since $p$ was arbitrary, we have $\tau(A)=\infty$ as required. 
The proof of (4) is analogous to that of (3), and (5) follows immediately from (3).

We concentrate on the ideal $\mathcal{T}_{f h}$, but we note that the ideals

$$
\mathcal{T}_{f, h, n}=\operatorname{Exh}\left(\tau_{n}\right)=\left\{A: \lim _{i} \tau_{n}(A \backslash i)=0\right\}, \quad n \in \mathbb{N},
$$

can turn out to be interesting in their own right. All these are P-ideals which are not $F_{\sigma}$ (assuming they are proper ideals, of course), since a mapping witnessing $\emptyset \times$ Fin $\leq_{\mathrm{RB}} \mathcal{T}_{f, h, n}$ can be easily obtained from an $(n-1)$-good sequence $($ see $\S 6$ ).

4. Properties of Tsirelson submeasures. In this section we show several lemmas which will be used in the proof of Theorem 3.1. First we give a transparent description of how $\tau_{n}$ is computed in Lemma 4.2 below. By $\mathbb{N}<\mathbb{N}$ we denote the set of all finite sequences of integers, and consider it as a tree under the ordering of end-extension. A set $T \subseteq \mathbb{N}^{<\mathbb{N}}$ is a tree if it is closed under taking initial segments of its elements. Note that the height of a finite tree $T$ is equal to the maximal length of its elements. By \langle\rangle we denote the empty sequence in $\mathbb{N}<\mathbb{N}$, and $t^{\wedge} i$ is the sequence obtained by concatenating $t$ with $\langle i\rangle$. An end-node of $T$ is any $t \in T$ such that $t^{\wedge} i \notin T$ for all $i$. (Note that end-nodes of $T$ do not necessarily belong to its top level.)

Definition 4.1. A family $\left\langle E_{t}: t \in T\right\rangle$ is an $h$-tree if $T \subseteq \mathbb{N}<\mathbb{N}$ is a finitely branching finite tree, the sets $E_{t}$ are finite, and for all $t \in T$, if $t^{\wedge} 1, \ldots, t^{\wedge} l$ are the immediate successors of $t$ in $T$ then

(1) $E_{t^{\wedge} 1}<\ldots<E_{t^{\wedge} l}$ and $E_{t}=\bigcup_{i=1}^{l} E_{t^{\wedge} l}$,

(2) $l \leq h\left(\min E_{t^{\wedge} 1}\right)$, i.e. $\left\langle\min E_{t^{\wedge} 1}, \ldots, E_{t^{\wedge} l}\right\rangle$ is $h$-admissible, and

(3) if $t$ is an end-node of $T$, then $E_{t}$ is a singleton.

The height of $\left\langle E_{t}: t \in T\right\rangle$ is the height of $T$. Note that every $i \in E_{\langle\rangle}$ defines a unique branch,

$$
B_{i}=\left\{t \in T: i \in E_{t}\right\},
$$

of $T$. The length, $\left|B_{i}\right|$, of this branch is equal to the length of its last node. A function $g: \mathbb{N} \rightarrow\{0,1, \ldots, n, \infty\}$ is an $(h, n)$-weight assignment if there is an $h$-tree $\left\langle E_{t}: t \in T\right\rangle$ of height at most $n$ such that

(4) $E_{\langle\rangle}=\{i: g(i) \neq \infty\}$,

(5) $g(i)=\left|B_{i}\right|$ for each $i \in E_{\langle\rangle}$.

Lemma 4.2. Assume that $h: \mathbb{N} \rightarrow \mathbb{N}$ is strictly increasing. Then for every s we have

$$
\tau_{n}(s)=\sup _{g} \sum_{i \in s} 2^{-g(i)} f(i),
$$

where the supremum is taken over all $(h, n)$-weight assignments $g$. 
Proof. Note that every branching of an $h$-tree corresponds to an application of a step in the recursive definition of $\tau_{n+1}$, and that the nodes of height $k$ come with the weight equal to $2^{-k}$ because of the $k$-fold multiplication with $1 / 2$. Condition (3) corresponds to $\tau_{0}(A)=\sup _{i \in A} f(i)$. Therefore, the lemma is proved by a straightforward induction on $n$.

LEMma 4.3. If $f, h$ are as in Lemma $4.2, n \in \mathbb{N}$ and $s$ is finite, then there is an $s^{\prime} \subseteq s$ such that $\tau_{n}\left(s^{\prime}\right)=\tau_{n}(s)$ and $\tau\left(s^{\prime}\right) \leq 3 \tau_{n}\left(s^{\prime}\right) / 2$.

Proof. Since $s$ is finite, the supremum appearing in Lemma 4.2 is attained for some $(h, n)$-weight assignment $g$; let $s^{\prime}=\{i: g(i) \neq \infty\} \cap s$. It suffices to prove that $\tau_{m}\left(s^{\prime}\right) \leq 3 \tau_{n}\left(s^{\prime}\right) / 2$ for every $m$. Fix $m$, let $g_{m}$ be some $(h, m)$-weight assignment, and let $X=\left\{i \in s^{\prime}: g_{m}(i)>g(i)\right\}$ and $Y=\left\{i \in s^{\prime}: g_{m}(i) \leq g(i)\right\}$. We claim that

$$
\sum_{i \in Y} 2^{-g_{m}(i)} f(i) \leq \tau_{n}\left(s^{\prime}\right) .
$$

To verify this, by Lemma 4.2 it suffices to show that the map $g^{\prime}$ defined by

$$
g^{\prime}(i)= \begin{cases}g_{m}(i) & \text { if } g_{m}(i) \leq g(i), \\ \infty & \text { if } g_{m}(i)>g(i) \text { or } g_{m}(i)=\infty,\end{cases}
$$

is an $(h, n)$-weight assignment. To see this, let $\left\langle E_{t}: t \in T\right\rangle$ be an $h$-tree of height $m$ witnessing that $g_{m}$ is an $(h, m)$-weight assignment. Then for $T^{\prime}=\{t \in T:|t| \leq n\}$ the family $\left\langle E_{t} \cap\left\{i: g^{\prime}(i) \neq \infty\right\}: t \in T^{\prime}\right\rangle$ is an $h$-tree (this follows immediately from the definitions). Since $g$ is an $(h, n)$-weight assignment, this tree is of height at most $n$ and it witnesses that $g_{m}^{\prime}$ is an $(h, n)$-weight assignment.

Therefore $(\dagger)$ is true and since $X \subseteq s^{\prime}$ we have

$$
\begin{aligned}
\tau_{m}\left(s^{\prime}\right) & =\sum_{i \in X} 2^{-g_{m}(i)} f(i)+\sum_{i \in Y} 2^{-g_{m}(i)} f(i) \\
& \leq \frac{1}{2} \sum_{i \in X} 2^{-g(i)} f(i)+\tau_{n}\left(s^{\prime}\right) \leq \frac{1}{2} \tau_{n}\left(s^{\prime}\right)+\tau_{n}\left(s^{\prime}\right) .
\end{aligned}
$$

Since $m$ was arbitrary and $\tau\left(s^{\prime}\right)=\sup _{m} \tau_{m}\left(s^{\prime}\right)$, this concludes the proof.

Recall that $\nu_{f}(s)=\sum_{i \in S} f(i)$.

Lemma 4.4. Assume $\lim _{n} f(n)=0$ and $h: \mathbb{N} \rightarrow \mathbb{N}$ is strictly increasing. Then for all $s$ and $n$ we have $\nu_{f}(s) \geq 2^{n+1}\left(\tau_{n+1}(s)-\tau_{n}(s)\right)$.

Proof. Fix an $\varepsilon>0$. Let $g$ be an $(h, n+1)$-weight assignment such that

$$
\sum_{i \in s} 2^{-g(i)} f(i) \geq \tau_{n+1}(s)+\varepsilon,
$$

as given by Lemma 4.2. Let $s_{0}=\{i \in s: g(i) \leq n\}$. Then by a weight assignment argument identical to that in the proof of Lemma 4.3 we have 
$\tau_{n}(s) \geq \tau_{n}\left(s_{0}\right) \geq \sum_{i \in s_{0}} 2^{-g(i)} f(i)$, and therefore

$$
\begin{aligned}
2^{-(n+1)} \nu_{f}(s) & \geq 2^{-(n+1)} \sum_{i \in s \backslash s_{0}} f(i) \\
& \geq \sum_{i \in s} 2^{-g(i)} f(i)-\sum_{i \in s_{0}} 2^{-g(i)} f(i) \geq \tau_{n+1}(s)-\tau_{n}(s)+\varepsilon .
\end{aligned}
$$

Since $\varepsilon>0$ was arbitrary, this concludes the proof.

Recall that if $\left\{w_{i}\right\}$ is a sequence of sets and $A \subseteq \mathbb{N}$, then we write $w_{A}=\bigcup_{i \in A} w_{i}$. The following lemma will be very useful (recall the definition of $\tau_{n}^{k}$ from $\left.\S 3\right)$.

Lemma 4.5. Assume $f, h$ are as in Lemma $4.2, w_{1}<w_{2}<\ldots$ is a sequence of finite sets, and $\delta>0$. If for all $i$ we have

(1) $\tau_{n}\left(w_{i}\right)<\delta / 2$, and

(2) $\tau_{n-1}^{\max \left(w_{i}\right)}\left(w_{[i+1, \infty)}\right)<\delta\left(\right.$ taking $\left.\max \left(w_{0}\right)=1\right)$,

then for every $A \subseteq \bigcup_{i} w_{i}$ we have $\tau_{n}(A)<\delta$.

Proof. If $\tau_{n}(A)=\tau_{n-1}(A)$, the conclusion follows from (2) above.

Claim 4.6. Under the above assumptions, if $A \subseteq \bigcup_{i} w_{i}$ and $\tau_{n}(A)>$ $\tau_{n-1}(A)$, then

$$
\tau_{n}(A) \leq \sup _{i}\left(\tau_{n}\left(w_{i}\right)+\frac{1}{2} \tau_{n-1}^{\max } w_{i}\left(\bigcup_{j=i+1}^{\infty} w_{j}\right)\right) .
$$

Proof. Fix $\varepsilon>0$. By the assumption, we have

$$
\tau_{n}(A) \leq \frac{1}{2} \sum_{j=1}^{m} \tau_{n-1}\left(E_{j} A\right)+\varepsilon
$$

for some $m \leq h(k)$ and $k<E_{1}<\ldots<E_{m}$. Let $i$ be the minimal such that $k \leq \max w_{i}$. If $\max E_{l} \leq \max w_{i}$ for all $l=1, \ldots, h(k)$, then $\tau_{n}(A) \leq$ $\tau_{n}\left(w_{i}\right)+\varepsilon$, and there is nothing to prove. Let $l$ be the minimal such that $\max E_{l}>\max w_{i}$. Then

$$
\begin{aligned}
\tau_{n}(A) \leq & \frac{1}{2} \sum_{j=1}^{l-1} \tau_{n-1}\left(E_{j} A\right)+\tau_{n-1}\left(E_{l} A\right)+\frac{1}{2} \sum_{j=l}^{m} \tau_{n-1}\left(E_{j} A\right)+\varepsilon \\
\leq & \frac{1}{2} \sum_{j=1}^{l-1} \tau_{n-1}\left(E_{j} A\right)+\tau_{n-1}\left(E_{l}\left(A w_{i}\right)\right) \\
& +\tau_{n-1}\left(E_{l}\left(A \backslash w_{i}\right)\right)+\frac{1}{2} \sum_{j=l}^{m} \tau_{n-1}\left(E_{j} A\right)+\varepsilon
\end{aligned}
$$




$$
\leq \tau_{n}\left(w_{i}\right)+\frac{1}{2} \tau_{n-1}^{k}\left(\bigcup_{p=i+1}^{\infty} w_{p}\right)+\varepsilon .
$$

Since $k \leq \max w_{i}$ and $\varepsilon>0$ was arbitrarily small, this completes the proof.

Lemma 4.5 follows immediately by the claim.

5. The second reduction. The main result of this section is Proposition 5.3, essentially saying that if $\mathcal{I} \leq_{\mathrm{RB}} \mathcal{T}_{f h}$ then $\mathcal{I}$ is of the form $\mathcal{T}_{f^{\prime} h^{\prime}}$ for some $f^{\prime}, h^{\prime}$ (possibly with $\lim _{i} f^{\prime}(i) \neq 0$ ). It is essentially due to Casazza, Johnson and Tzafriri (see [1] and [2, Proposition I.12 and Lemmas II.1 and II.3]), who used the case when $h$ is the identity function, to prove that every infinite-dimensional subspace of Tsirelson space includes a copy of Tsirelson space. We reproduce the proof from [1] for the convenience of the reader.

Lemma 5.1. Assume $f: \mathbb{N} \rightarrow \mathbb{R}$ is nonnegative and $h: \mathbb{N} \rightarrow \mathbb{N}$ is strictly increasing, and let $h^{+}(n)=h(n+h(n))$. Then for every $A$ and $n$ we have

$$
\tau_{f, h, n}(A) \leq \tau_{f, h^{+}, n}(A) \leq 3 \tau_{f, h, n}(A) .
$$

Pr o of. The left-hand side inequality is obvious, since $h^{+} \geq h$. We prove the following strengthening of the right-hand side inequality by induction:

(*) For all $A$ and $n$ there are sets $F_{1}<F_{2}<F_{3}$ such that

$$
\tau_{f, h^{+}, n}(A) \leq \sum_{j=1}^{3} \tau_{f, h, n}\left(F_{j} A\right) .
$$

The case when $n=0$ is trivial, so let us assume the lemma is proved for some $n$ and prove it for $n+1$. If $\tau_{f, h^{+}, n+1}(A)=\tau_{f, h^{+}, n}(A)$, then there is nothing to prove, so we can assume

$$
\begin{aligned}
\tau_{f, h^{+}, n+1}(A) & =\frac{1}{2} \sum_{l=1}^{h^{+}(k)} \tau_{f, h^{+}, n}\left(E_{l} A\right) \quad \text { for some } h^{+} \text {-admissible }\langle k, \vec{E}\rangle, \\
& \leq \frac{1}{2} \sum_{l=1}^{h^{+}(k)} \sum_{j=1}^{3} \tau_{f, h, n}\left(F_{l j} E_{l} A\right) \quad \text { for some } F_{l 1}<F_{l 2}<F_{l 3} .
\end{aligned}
$$

We can assume $F_{l j} E_{l}=F_{l j}$ for all $l, j$. For $G_{3(l-1)+j}=F_{l j}$ we have $G_{1}<$ $G_{2}<\ldots<G_{3 h^{+}(k)}$. We can assume all $G_{l}$ 's are nonempty, possibly by eliminating the empty ones from the sequence. Let $k^{*}=k+h(k)$ (so that $\left.h^{+}(k)=h\left(k^{*}\right)\right)$ and

$$
F_{1}=\bigcup_{l=1}^{h(k)} G_{l}, \quad F_{2}=\bigcup_{l=h(k)+1}^{h(k)+h\left(k^{*}\right)} G_{l}, \quad F_{3}=\bigcup_{l=h(k)+h\left(k^{*}\right)+1}^{3 h^{+}(k)} G_{l} .
$$


Then $\left\langle k, G_{1}, \ldots, G_{h(k)}\right\rangle$ is $h$-admissible, so we have

$$
\tau_{f, h, n+1}\left(F_{1} A\right) \geq \frac{1}{2} \sum_{l=1}^{h(k)} \tau_{f, h, n}\left(G_{l} A\right) .
$$

Note that, since each $G_{l}$ is nonempty, we have $\min \left(G_{h(k)+1}\right) \geq \min G_{1}+$ $h(k) \geq k^{*}$, and therefore the tuple associated with $F_{2}$ is $h$-admissible and we have

$$
\tau_{f, h, n+1}\left(F_{2} A\right) \geq \frac{1}{2} \sum_{l=h(k)+1}^{h\left(k^{*}\right)} \tau_{f, h, n}\left(G_{l} A\right) .
$$

Like before, $\min G_{h(k)+h\left(k^{*}\right)+1} \geq k^{*}+h\left(k^{*}\right)$. Since $k \leq h(k)$ and $h(2 k) \leq$ $h\left(k^{*}\right)$, we have (note that $h(i)+j \leq h(i+j)$, since $h$ is strictly increasing)

$$
3 h^{+}(k) \leq h\left(k^{*}\right)+2 h\left(k^{*}\right) \leq h\left(k^{*}\right)+h\left(k^{*}+h\left(k^{*}\right)\right)
$$

and $3 h^{+}(k)-h\left(k^{*}\right) \leq h\left(k^{*}+h\left(k^{*}\right)\right)$, so the tuple associated with $F_{3}$ is $h$-admissible, and we have

$$
\tau_{f, h^{+}, n+1}(A) \leq \frac{1}{2} \sum_{l=1}^{3 h(2 k))} \tau_{f, h, n}\left(G_{l} A\right) \leq \sum_{j=1}^{3} \tau_{f, h, n+1}\left(F_{j} A\right),
$$

completing the inductive proof.

Lemma 5.2. Assume $f, h$ are as in Lemma 5.1 and that $w_{1}<w_{2}<\ldots$ are finite sets. Let $f^{\prime}(i)=\tau_{f, h}\left(w_{i}\right), h^{\prime}(i)=h\left(\min w_{i}\right)$, and $h^{\prime \prime}(i)=h\left(\max w_{i}\right)$. Then for all $A \subseteq \mathbb{N}$ we have

$$
\tau_{f^{\prime}, h^{\prime}}(A) \leq \tau_{f, h}\left(w_{A}\right) \leq 6 \tau_{f^{\prime}, h^{\prime \prime}}(A) .
$$

Pr o of. We first prove the left-hand side inequality, by proving

$$
\tau_{f^{\prime}, h^{\prime}, n}(A) \leq \tau_{f, h}\left(w_{A}\right)
$$

using induction on $n$. Since $\tau_{f^{\prime}, h^{\prime}}(A)=\lim _{n} \tau_{f^{\prime}, h^{\prime}, n}(A)$, this will suffice. In the case when $n=0$ for some $i \in A$ we have $\tau_{f^{\prime}, h^{\prime}, 0}(A)=\tau_{f, h}\left(w_{i}\right) \leq$ $\tau_{f, h}\left(\bigcup_{j \in A} w_{j}\right)$.

Now assume $(*)$ is true for $n$. If $\tau_{f^{\prime}, h^{\prime}, n+1}(A)=\tau_{f^{\prime}, h^{\prime}, n}(A)$, there is nothing to prove. So we can assume

$$
\tau_{f^{\prime}, h^{\prime}, n+1}(A)=\frac{1}{2} \sum_{j=1}^{h^{\prime}(k)} \tau_{f^{\prime}, h^{\prime}, n}\left(E_{j} A\right) \quad \text { for some } h \text {-admissible }\langle k, \vec{E}\rangle .
$$

(Assuming that $|\vec{E}|=h^{\prime}(k)$ is clearly not a loss of generality.) Since $h^{\prime}$ is increasing, we can assume $k=\min E_{1}$, and therefore $h^{\prime}\left(\min E_{1}\right)=h\left(\min w_{k}\right)$. Let $E_{j}^{\prime}=\bigcup_{j \in E_{i}} w_{j}$. Then $\left\langle\min w_{k}, E_{1}^{\prime}, \ldots, E_{h^{\prime}(k)}^{\prime}\right\rangle$ is $h$-admissible, by the 
inductive assumption we have

$$
\frac{1}{2} \sum_{j=1}^{h^{\prime}(k)} \tau_{f^{\prime}, h^{\prime}, n}\left(E_{j} A\right) \leq \frac{1}{2} \sum_{j=1}^{h(\min w(k))} \tau_{f, h}\left(E_{j}^{\prime} w_{A}\right) \leq \tau_{f, h}\left(w_{A}\right),
$$

and this ends the verification of the left-hand side inequality.

Now we prove the right-hand side inequality. Let $h^{*}(i)=2 h^{\prime \prime}(i)$. Since $2 h^{\prime \prime}(i) \leq h^{\prime \prime}\left(i+h^{\prime \prime}(i)\right)$, Lemma 5.1 implies $\tau_{f^{\prime}, h^{*}, n} \leq 3 \tau_{f^{\prime} h^{\prime \prime}, n}$ for all $n$, and therefore it suffices to prove

$$
\tau_{f, h, n}\left(w_{A}\right) \leq 2 \tau_{f^{\prime}, h^{*}, n}(A)
$$

using induction on $n$. When $n=0$ for some $i \in A$ we have

$$
\tau_{f, h, 0}\left(w_{A}\right)=\tau_{f, h, 0}\left(w_{i}\right) \leq \tau_{f, h}\left(w_{i}\right)=\tau_{f^{\prime}, h^{\prime \prime}, 0}(i) \leq 2 \tau_{f^{\prime}, h^{*}, 0}(A) .
$$

Now we assume the lemma is true for $n$ and prove it for $n+1$. Again we can assume that $\tau_{f, h, n+1}\left(w_{A}\right)>\tau_{f, h, n}\left(w_{A}\right)$, therefore for some $h$-admissible $\langle k, \vec{E}\rangle$ (without loss of generality, we can assume that $k=\min E_{1}$ and $|\vec{E}|=h(k))$ we have

$$
\tau_{f, h, n+1}\left(w_{A}\right)=\frac{1}{2} \sum_{l=1}^{h(k)} \tau_{f, h, n}\left(E_{l} w_{A}\right) .
$$

We can assume $w_{A} \subseteq \bigcup_{l=1}^{h(k)} E_{l}$. Let $E_{l}^{-}=\left\{i: \min \left(w_{i}\right) \in E_{l}\right\}$ and $E_{l}^{+}=\{i$ : $\left.\max \left(w_{i}\right) \in E_{l}\right\}$. Then by the inductive assumption

$$
\begin{aligned}
\frac{1}{2} \sum_{l=1}^{h(k)} \tau_{f, h, n}\left(E_{l} w_{A}\right) & \leq \frac{1}{2} \sum_{l=1}^{h(k)} 2 \tau_{f, h, n}\left(E_{l}^{+} A\right)+\frac{1}{2} \sum_{l=1}^{h(k)} 2 \tau_{f, h, n}\left(E_{l}^{-} A\right) \\
& \leq \frac{1}{2} \sum_{l=1}^{h(k)} 2 \tau_{f^{\prime}, h^{*}, n}\left(E_{l}^{+} A\right)+\frac{1}{2} \sum_{l=1}^{h(k)} 2 \tau_{f^{\prime}, h^{*}, n}\left(E_{l}^{-} A\right) .
\end{aligned}
$$

If $j=\min \left(\bigcup_{l=1}^{h(k)}\left(E_{l}^{+} \cup E_{l}^{-}\right)\right)$, then $h^{\prime \prime}(j)=h\left(\max w_{j}\right) \geq h\left(\min E_{1}\right)=h(k)$, also $h^{*}(j)=2 h^{\prime \prime}(j) \geq 2 h(k)$, so that $\left\langle j, \vec{E}^{*}\right\rangle$ (where $\vec{E}^{*}$ is the increasing enumeration of $\left.\bigcup_{l=1}^{h(k)}\left(E_{l}^{-} \cup E_{l}^{+}\right)\right)$is $h^{*}$-admissible, so that the right-hand side is equal to at most $2 \tau_{f^{\prime}, h^{*}, n+1}(A)$. As pointed out earlier, this concludes the proof since $\tau_{f^{\prime}, h^{*}, n+1} \leq \tau_{f^{\prime}, h^{\prime \prime}, n+1}$ for all $n$.

We are now prepared for the main result of this section.

Proposition 5.3. Assume $f: \mathbb{N} \rightarrow \mathbb{R}$ is nonnegative and $h: \mathbb{N} \rightarrow \mathbb{N}$ is strictly increasing. If $w_{1}<w_{2}<\ldots$ are finite sets, then for $f^{\prime}(i)=\tau_{f, h}\left(w_{i}\right)$ and $h^{\prime}(i)=h\left(\min w_{i}\right)$ we have $\mathcal{T}_{f^{\prime}, h^{\prime}}=\left\{A: w_{A} \in \mathcal{T}_{f h}\right\}$. 
Proof. Let $h^{\prime \prime}(i)=\max \left(w_{i}\right)$ and $\left(h^{\prime}\right)^{+}(i)=h^{\prime}\left(i+h^{\prime}(i)\right)$. Since $h^{\prime \prime} \leq$ $\left(h^{\prime}\right)^{+}$, Lemmas 5.1 and 5.2 imply that for every $A$ we have

$$
\tau_{f^{\prime}, h^{\prime}}(A) \leq \tau_{f, h}\left(w_{A}\right) \leq 6 \tau_{f^{\prime}, h^{\prime \prime}}(A) \leq 6 \tau_{f^{\prime}\left(h^{\prime}\right)+}(A) \leq 18 \tau_{f^{\prime}, h^{\prime}}(A),
$$

thus $\tau_{f, h}\left(w_{A}\right)=\infty$ if and only if $\tau_{f^{\prime}, h^{\prime}}(A)=\infty$, and the two ideals coincide.

6. Good sequences. An important part of the proof of Theorem 3.1 is in proving its weaker version:

Proposition 6.1. If $h$ is strictly increasing, the ideals $\mathcal{T}_{f h}$ and $\mathcal{I}_{1 / n}$ are different.

Proof. Assume the contrary, that $\mathcal{T}_{f h}=\mathcal{I}_{1 / n}$. Note that we can assume $\lim _{i} f(i)=0$, for otherwise there would be an infinite set $A$ none of whose infinite subsets is in $\mathcal{T}_{f h}$, but there is no such set for $\mathcal{I}_{1 / n}$.

Claim 6.2. There is a sequence $t_{1}<t_{2}<\ldots$ and $N \in \mathbb{N}$ such that

(a) $\nu_{f} \leq N \nu_{1 / n}$ on $\bigcup_{i} t_{i}$, and

(b) $\inf _{i}\left(\tau_{1}\left(t_{i}\right)\right)>0$.

Proof. Let $I_{m}=[m, m+h(m))$ and note that $\tau\left(I_{m}\right)=\tau_{1}\left(I_{m}\right) / 2=$ $\nu_{f}\left(I_{m}\right) / 2$. We shall prove that there is an $N \in \mathbb{N}$ such that if $t_{m}^{0}=\{k \in$ $\left.I_{m}: k f(k)<N\right\}$, then all but finitely many $t_{m}^{0}$ satisfy $\nu_{1 / n}\left(t_{m}^{0}\right) \geq(\ln 2) / 2$. If we can find such a sequence, then we can take $t_{i}$ to be its subsequence satisfying $t_{1}<t_{2}<\ldots$, and (a) will be satisfied. To assure (b), note that $\liminf _{i} \nu_{f}\left(t_{i}\right)>0$, since otherwise there would be an infinite $C \subseteq \mathbb{N}$ such that $Y=\bigcup_{i \in C} t_{i}$ satisfies $\nu_{f}(Y)<\infty$, but then $Y \in \mathcal{T}_{f h} \backslash \mathcal{I}_{1 / n}$, contradicting our assumptions. Therefore we can find a subsequence of $t_{i}$ which satisfies $\inf _{i} \nu_{f}\left(t_{i}\right)>0$, and since $t_{i} \subseteq\left[m_{i}, m_{i}+h\left(m_{i}\right)\right)$ for some $m_{i}$, this implies (b).

Assume that $N$ as above does not exist. Then we can find $\{m(N)\}_{N=1}^{\infty}$ satisfying

(1) $\nu_{1 / n}\left\{k \in I_{m(N)}: k f(k)<2^{N}\right\}<(\ln 2) / 2$,

(2) $k>2^{N}$ for all $k \in I_{m(N)}$ with $f(k)<2^{-N}$, and

(3) the intervals $I_{m(N)}$ are pairwise disjoint.

Since $\nu_{1 / n}\left(I_{m}\right) \geq \ln (m+h(m))-\ln (m) \geq \ln 2$ for all $m$, by (1) we have

$$
\nu_{1 / n}\left\{k \in I_{m(N)}: k f(k) \geq 2^{N}\right\}>(\ln 2) / 2 .
$$

Let $s_{N} \subseteq I_{m(N)}$ be such that

(4) $k f(k) \geq 2^{N}$ for all $k \in s_{N}$, and

(5) $\left|\nu_{1 / n}\left(s_{N}\right)-2^{-N+1}\right|<2^{-N}$.

(Note that (5) can be assured by using (2).) Then we have

$$
\tau\left(s_{N}\right)=\nu_{f}\left(s_{N}\right) / 2 \geq 2^{N} \nu_{1 / n}\left(s_{N}\right) \geq 2^{N} \cdot 2^{-N}=1,
$$


therefore $\bigcup_{N} s_{N} \in \mathcal{I}_{1 / n} \backslash \mathcal{T}_{f h}$, contradicting our assumption and completing the proof of Claim 6.2.

We will use $\left\{t_{i}\right\}$ given by Claim 6.2 to find $\left\{w_{j}\right\}$ such that for all $j$,

(6) $w_{1}<w_{2}<\ldots$ are included in $\bigcup_{i} t_{i}$,

(7) $\tau_{j+1}\left(w_{j}\right) \geq 2^{-j+1}$, and

(8) $\tau_{j}\left(w_{j}\right) \leq 2^{-j}$.

Assume $\left\{w_{j}\right\}$ satisfy (6)-(8) above, and find $v_{j} \subseteq w_{j}$ such that $\tau\left(v_{j}\right) \leq$ $3 \tau_{j+1}\left(w_{j}\right) / 2$ and $\tau_{j+1}\left(v_{j}\right)=\tau_{j+1}\left(w_{j}\right)$. Therefore $\tau\left(v_{j}\right)<2^{-j+2}$ and by Lemma 4.4 we have $\nu_{f}\left(v_{j}\right) \geq 2^{j+1}\left(2^{-j+1}-2^{-j}\right)=2$. Note that $\tau \leq \nu_{f}$ and $\nu_{f} \leq N \nu_{1 / n}$ on the set $X=\bigcup_{j} v_{j} \subseteq \bigcup t_{i}$, and therefore $\lim _{n} \tau(X \backslash n)<\infty$ and $\lim _{n} \nu_{f}(X \backslash n)=\infty$. This implies $X \in \mathcal{T}_{f h} \backslash \mathcal{I}_{f} \subseteq \mathcal{I}_{f h} \backslash \mathcal{I}_{1 / n}$, contradicting our assumptions.

Therefore it suffices to find $\left\{w_{i}\right\}$ satisfying (6)-(8) above. We do this by using the following notion (recall that $u_{A}=\bigcup_{i \in A} u_{i}$ ).

Definition 6.3. A sequence $u_{1}<u_{2}<\ldots$ of finite sets is $m$-good for $f, h$ (or simply $m$-good if $f, h$ are clear from the context) if there exists a $\delta>0$ such that:

(i) the set $\left\{\tau_{m}\left(u_{i}\right): i \in \mathbb{N}\right\}$ is dense in $[0, \delta]$, and

(ii) $\lim _{i} \tau_{m-1}\left(u_{[i, \infty)}\right)=0$.

Lemma 6.4. Assume $\lim _{n} f(n)=0$ and $h$ is strictly increasing. If a sequence $\left\{u_{i}\right\}$ is m-good and $a, \varepsilon>0$, then there is a finite $v \subseteq \bigcup_{i} u_{i}$ satisfying $\left|\tau_{m+1}(v)-a\right|<\varepsilon$ and $\tau_{m}(v)<\varepsilon$.

Proof. We can assume that $\varepsilon<\delta$, where $\delta$ is as in the definition of good sequence. By going to a subsequence we can also assume that for all $i$ we have

$$
\left.\tau_{m-1}^{\max u_{i}}\left(u_{[i+1, \infty)}\right)<\varepsilon \quad \text { (taking } \max \left(u_{0}\right)=1\right) .
$$

The set $A=\left\{i: \tau_{m}\left(u_{i}\right)<\varepsilon / 2\right\}$ is infinite and $\lim \sup _{k \in A} \tau_{m}\left(u_{k}\right)=\varepsilon / 2$ (because the sequence $\left\{u_{i}\right\}$ is $m$-good and the corresponding $\delta$ is bigger than $\varepsilon / 2)$. By Lemma 3.2(4) we have $\tau_{m+1}\left(u_{A}\right)=\infty$. Therefore we can find $v \subseteq u_{A}$ such that $\tau_{m+1}(v) \geq a, \tau_{m+1}\left(v^{\prime}\right)<a$ for every $v^{\prime} \subsetneq v$, and $f(j)<\varepsilon$ for every $j \in v$. Then by the subadditivity of $\tau_{m+1}$ we have $\left|\tau_{m+1}(v)-a\right|=\tau_{m+1}(v)-a<\varepsilon$. By Lemma 4.5 (applied with $\delta=\varepsilon$ ) we have $\tau_{m}(v)<\varepsilon$, therefore $v$ is as required.

Lemma 6.5. Assume $\lim _{n} f(n)=0$ and $h$ is strictly increasing. Then for every $m$-good sequence $\left\{u_{i}\right\}$ there is an $(m+1)$-good sequence $\left\{v_{i}\right\}$ such that $\bigcup_{i} v_{i} \subseteq \bigcup_{i} u_{i}$.

Proof. Let $q_{i}(i \in \mathbb{N})$ be an enumeration of all rationals in the interval $(0,1)$. By using Lemma 6.4, we can recursively find $v_{1}<v_{2}<\ldots$ included in 
$\bigcup_{i} u_{i}$ and such that $\left|\tau_{m+1}\left(v_{i}\right)-q_{i}\right|<2^{-i}$ and $\tau_{m}\left(v_{i}\right)<2^{-i}$. Then for every $i$ we have $\tau_{m}\left(v_{[i, \infty)}\right)<2^{-i+1}$, therefore the sequence $v_{i}$ is $(m+1)$-good.

Let $t_{i}$ be as in Claim 6.2, and let $\varepsilon=\inf _{i} \tau_{1}\left(t_{i}\right)>0$. Since $\lim _{n} f(n)=0$, we can find $u_{i} \subseteq t_{i}(i \in \mathbb{N})$ so that $\left\{\tau_{1}\left(u_{i}\right): i \in \mathbb{N}\right\}$ is dense in $[0, \varepsilon]$. Since the condition $\lim _{i} \tau_{0}\left(u_{i}\right)=0$ reduces to our assumption that $\lim _{k} f(k)=0$, this sequence is 1 -good. Therefore the sequence $w_{i}$ as in (6)-(8) can now be constructed recursively by using Lemmas 6.5 and 6.4. As explained before, this implies that the two ideals differ and concludes the proof of Proposition 6.1 .

The above proof gives the following proposition of independent interest (see the proof of [5, Proposition 3.6]).

Proposition 6.6. If $\lim _{n} f(n)=0, h: \mathbb{N} \rightarrow \mathbb{N}$ is strictly increasing and

$$
\liminf _{n} \nu_{f}([n, n+h(n))>0 \text {, }
$$

then for every $m \geq 1$ there is an $m$-good sequence.

7. Proof of Theorem 3.1. The ideal $\mathcal{T}_{f h}$ is, by Lemma 3.2(5), an $F_{\sigma}$ P-ideal. Therefore it remains to prove that $\mathcal{P}(\mathbb{N}) / \mathcal{I}_{1 / n} \mathbb{Z}_{\mathrm{B}} \mathcal{P}(\mathbb{N}) / \mathcal{T}_{f h}$. By Lemma 2.1, it suffices to prove that there is no sequence of finite sets $w_{1}<w_{2}<\ldots$ such that for all $A \subseteq \mathbb{N}$ we have

$$
A \in \mathcal{I}_{1 / n} \text { if and only if } \bigcup_{i \in A} w_{i} \in \mathcal{T}_{f h} .
$$

Assume that such a sequence exists. By Proposition 5.3, for some strictly increasing $h^{\prime}$ and $f^{\prime}(n)=\tau_{f, h}\left(w_{n}\right)$ the ideals $\mathcal{I}_{1 / n}$ and $\mathcal{T}_{f^{\prime} h^{\prime}}$ coincide, but this contradicts Proposition 6.1 and completes the proof.

\section{References}

[1] P. G. Casazza, W. B. Johnson and L. Tzafriri, On Tsirelson's space, Israel J. Math. 47 (1984), 81-98.

[2] P. G. Casazza and T. J. Shura, Tsirelson's Space, Lecture Notes in Math. 1363, Springer, 1980.

[3] I. Farah, Analytic quotients, to appear.

[4] -, Analytic ideals and their quotients, PhD thesis, University of Toronto, 1997.

[5] -, Basis problem for turbulent actions, preprint, 1998.

[6] W. T. Gowers, Recent results in the theory of infinite-dimensional Banach spaces, in: Proc. Internat. Congress of Mathematicians, Zürich 1994, Birkhäuser, 1995, 932-942.

[7] G. Hjorth, Actions by classical Banach spaces, J. Symbolic Logic, to appear.

[8] G. Hjorth and A. S. Kechris, New dichotomies for Borel equivalence relations, Bull. Symbolic Logic 3 (1997), 329-346. 
[9] A. S. Kechris, Rigidity properties of Borel ideals on the integers, Topology Appl. 85 (1998), 195-205.

[10] A. Louveau, On the size of quotients by definable equivalence relations, in: Proc. Internat. Congress of Mathematicians, Zürich 1994, Birkhäuser, 1995, 269-276.

[11] K. Mazur, $F_{\sigma}$-ideals and $\omega_{1} \omega_{1}^{*}$-gaps in the Boolean algebra $\mathcal{P}(\omega) / I$, Fund. Math. 138 (1991), 103-111.

[12] - Towards the dichotomy for $F_{\sigma}$-ideals, preprint, 1996.

[13] E. Odell and T. Schlumprecht, Distortion and stabilized structure in Banach spaces; New geometric phenomena for Banach and Hilbert spaces, in: Proc. Internat. Congress of Mathematicians, Zürich 1994, Birkhäuser, 1995, 955-965.

[14] M. R. Oliver, Borel upper bounds for the Louveau-Veličković and Mazur towers, preprint, 1998.

[15] S. Shelah, Proper Forcing, Lecture Notes in Math. 940, Springer, 1982.

[16] S. Solecki, personal communication, 1997.

[17] —, Analytic ideals, Bull. Symbolic Logic 2 (1996), 339-348.

[18] B. Veličković, Definable automorphisms of $\mathcal{P}(\omega) /$ fin, Proc. Amer. Math. Soc. 96 (1986), 130-135.

[19] -, A note on Tsirelson type ideals, Fund. Math., this issue.

Department of Mathematics

York University

North York, Ontario

Canada M3J 1P3

E-mail: ifarah@mathstat.yorku.ca
Matematički Institut

Kneza Mihaila 35

11000 Beograd

Yugoslavia 